\title{
PROJECTED GOVERNMENT NEEDS IN HUMAN LANGUAGE TECHNOLOGY AND THE ROLE OF RESEARCHERS IN MEETING THEM
}

\author{
Helen M. Gigley, Ph.D. \\ Head, Human Computer Interaction Laboratory \\ Naval Research Laboratory \\ CODE 5530 \\ Washington, D.C. $20375-5337$
}

\begin{abstract}
${ }^{1}$
After a brief discussion of current government uses and needs for human language technology, this paper will discuss the uses which will probably survive in the future as well as attempt to define some anticipated ones. Business uses, military uses, and education/training receive the most focus today. Current state of the art technologies are providing initial resources to enable better management of the above. Suggested critical efforts to make the technologies more appropriate for the tasks at hand in the government will be discussed. A tentative view of the role of researchers, in providing the basis to obtain adequate critical natural language technologies, will be presented.
\end{abstract}

\section{CURRENT GOVERNMENT NEEDS IN HUMAN LANGUAGE TECHNOLOGY}

The functions of government fall into three general classes:

1. business, both external and internal;

2. military, beyond the business uses; and

3. educational/training responsibilities.

One very important question related to determining current human language technology needs is to look at these three broad areas and to assess what technology exists today and how effectively it can be applied. Another equally important question which must be considered is, "Does technology help or hinder the functionality of the government?"

\subsection{Human Language Technology and Current Government Business Needs}

Government business needs are coextensive with operational business needs of the United States. Access to information, interpersonal communication, and joint work environments within government operations are all critical

1 The opinions and assertions in this paper are those of the author and are not to be construed as official or reflecting the views of the Department of the Navy. functional needs. Inter- and intra-agency coordination and information dissemination are critical to efficient and proper functioning of our government. When is natural language technology a suitable solution to maximize the effectiveness in these efforts? Some examples will shed light on the nature of our current human language technology capabilities and provide guidance on what still is needed even within our current government operations.

Information Management Functions of the Government:

- Access to personnel.

- Dissemination of information.

- Acquisition of information.

- Protection of access to information.

\section{Communication Functions of the Government:}

- External Communication Functions -need to communicate with the general public and provide suitable responses not only in content but in presentation style.

- Internal Communication Functions -communication of protocols, procedural modifications, relevant day to day operational changes.

* Multi-language interaction facility where the interaction may be in any of several languages as well as translation capabilities.

* Joint work environments where multiple decision making is enhanced via multitasking environments at distributed locations. 


\section{Education/Training Functions of the Government:}

- Required to maintain up-to-date knowledge of system operations and procedures.

- New personnel must be brought up to speed on how to function.

The bottom line for government-needed human language technology is that it should provide alternative resources for communication which increase the functional precision and response to enhance productivity.

\subsection{Human Language Technology and Current Military Needs}

Military human language technology needs include many of the logistical and business-type needs just mentioned. In addition, military needs include factors of real-time performance and secure access which are not absolute within business ones. The functions of military systems assume a different purpose. Presently, they also insist on a role for humans which many business functions attempt to minimize; these systems are Human-in-the-Loop Systems. Military systems enhance decision making, include dissemination of information having various forms, and often must work in real-time response arenas, under secure conditions. How to maximize the information capacity within Human-in-the-Loop decision support systems is a critical need. Human language technology has a vital part to play in providing this capability. Language capabilities need to function precisely, correctly, and efficiently in multiple modalities.

Military Decisions require real-time language facilities. Military decisions require integration of many types of information which exist in disparate forms. Language technologies provide a possible addition to current methods to increase the information available in stressful, short-response-time decision situations. A primary concern is to determine what language technologies to employ and under what conditions.

Security implications for human language technology appear chiefly as constraints on its use. Speech is available to all within range of its audible production. Its internal form may also be accessible. What language technologies maintain security and how might language technologies provide added secure access constraints? Voice recognition can be a key, but is it sufficient?

\subsection{Human Language Technology and Education / Training Needs}

Education and training needs in government function do not appear to be diminishing. Even though the work force might shrink, its demography is constantly changing. Meeting the challenge of developing suitably capable individuals from the current and future work force will greatly depend on how education needs for the entire U.S. are met.

Currently, demands on the government work force beyond physical plant maintenance and related in-house infrastructure function, minimally require language fluency in English. All positions require literacy in English. Second language fluency and literacy also have become a staffing criteria for some positions. All of these facts present problems for many individual's qualifications to work for the government.

Lack of language fluency and literacy will create a possible problem in the near future as older workers retire. Many of the tasks government workers perform are language based, whether accessing information, providing it, or collecting it. An issue is can we use technology to replace them? Do we want to? And if not, how will we guarantee that they can do the job? Training systems for language skills can be one viable solution. These systems will be multimodal and require all aspects of language function.

As an additional need for training and education, we will need to continually update the skills and facility of personnel on the job. This is a critical need in the military. Having language technology available to develop the training methodologies is critical. What will documents be like? How will one use the available language tools? Answers to these questions are unknown but are hinted at in the current multimode capabilities which can now be integrated on our workstations.

When considering education and training for the government, it should be the case that developed technology is also relevant and available to education systems throughout the country.

\section{CURRENT TECHNOLOGIES--ARE THEY HELPING OR HINDERING?}

As previously mentioned, successful application of technology can only be measured by its efficiency and precision in use. Otherwise we are in a state of technology only for technology's sake. Let's look at some currently available technologies which have been introduced into government. What can we learn from their introduction?

Immediately, one is struck by the fact that more often than not a technology is introduced and deployed publicly without any study as to its impact. We need to consider the impact of such adoptions before rather than after they get put into place. One only has to keep in mind the work of Gray, John, and Atwood (1) which demonstrated through analysis and data evaluation that introducing technology does not necessarily increase effectiveness. We are in that situation now with our language technologies. Caution is advised and careful scrutiny of the effects should be considered when adopting any new 
technological invention. A case in point is voice mail. My personal opinion is that it is one of the greatest cogs in U.S. competitiveness today.

Imagine yourself as a foreign business person calling a U.S. top company to place an order and having to sit for two minutes or more to reach the appropriate ordering department while menu options are read sequentially Often, in voice mail, one is not even sure the menu item selected is the appropriate one. The language technology relies on hardware selection which is sequential and numeric, but is this necessary?

We need to develop methods for studying the impact of potential technologies and their effect on function. We need to consider the problem from two viewpoints, the function being served within the government and equally important, the functional demands it places on nongovernment or citizen users of the technology even without their consent. Language technology more than other types of technology subtly conveys an institution's attitude toward its clientele. This interaction for many government functions is vital.

Functional transaction speed is an important factor of any technology that should always be considered. We need to minimize the impact of the technology on the efficiency of human functioning, and still be able to get necessary things done.

\section{ROLE OF RESEARCHERS IN MEETING LANGUAGE TECHNOLOGY NEEDS}

Researchers' primary focus continues to be studying basic scientific principles and using them to expand the capabilities of language technology regardless of modality. Evaluation and determination of constraints on applicability of the technology also need to be developed. Furthermore, researchers need not only to consider their contribution as a basic scientific result but must be aware of the potential use of the result within our society, both favorably and adversely.

Suppose many access functions are completely assigned to machines and that as a user, you need to obtain some vital information. When the machine responds that you are not permitted access even though you know you are, how will you be able to get to a responsible agent to correct the problem if you are talking to a machine? Will the responsible agent also be a machine? How will you find out it is a machine if all interactions are via language technology? Ultimately, with automatic language processes in place, how would you determine how to correct the situation? This is bordering on the question of reality and virtual environments and their detectability if and when we really produce language technologies that are indeterminable from real speakers. Is this possible?

Even now we can produce speech that sounds like a given individual based on sampling of the person's actual speech. Where will we draw the limits of such use? Where will be draw the limits of appropriateness for language technology usage in general? There are vital research issues to be addressed regarding these concerns.

\section{CONCLUSION}

Government needs for language technology encompass those of general business as well as more demanding military constraints. The technologies we currently have in place have not always been introduced expeditiously and beneficially. This does not mean that we should abandon all efforts to develop language, but instead points to a more cautionary view of accepting language technology as a solution. The basic efforts now underway will advance the role of language technology and will enhance functionality of government. We as researchers need to accept some of the responsibility for making sure the contributions to government function in the future remain as positive enhancements rather than harmful ones.

\section{REFERENCES}

1. Gray, Wayne D.; John, Bonnie E.; and Atwood, Michael E.; "The Precis of Project Emestine or An Overview of a Validation of GOMS," CHI 92 Conference Proceedings, Monterey, CA, pp. 307-312, 1992. 